\section{IMPLICACIONES DE LOS ESTUDIOS SOBRE MEMORIA COLECTIVA PARA LA ENSEÑANZA DE LA HISTORIA}

\author{
Maitane Arnoso \\ Universidad del País Vasco \\ ORCID iD: https://orcid.org/0000-0001-8606-749X \\ maitane_arnoso@yahoo.es \\ Darío Páez \\ Universidad del País Vasco \\ ORCID iD: https://orcid.org/0000-0002-8459-6037 \\ darioalexpaez@gmail.com \\ Magdalena Bobowik \\ Universidad del País Vasco \\ ORCID iD: https://orcid.org/0000-0002-2341-0665 \\ magdalena.bobowik@ehu.es \\ Nekane Basabe \\ Universidad del País Vasco \\ ORCID iD: https://orcid.org/0000-0003-4753-4299 \\ nekane.basabe@ehu.es
}

Cómo citar este artículo/Citation: Arnoso, M., Páez, D., Bobowik, M. y Basabe, N. (2018). Implicaciones de los estudios sobre memoria colectiva para la enseñanza de la historia. Arbor, 194 (788): a445. https://doi.org/10.3989/arbor.2018.788n2005

Recibido: 6 marzo 2015. Aceptado: 8 febrero 2016.

RESUMEN: La investigación sobre la memoria colectiva y las representaciones sociales de la historia en el campo de la psicología social puede proporcionar reflexiones de interés para el fortalecimiento de las competencias meta-cognitivas en la enseñanza de la historia. En este capítulo se revisa la bibliografía existente y la investigación empírica en este campo. En primer lugar, se analizan los procesos a través de los cuales se conforman las memorias y representaciones del pasado. A continuación, se exponen tres competencias de razonamiento necesarias para su aplicación en las prácticas de enseñanza: a) una centrada en la determinación de lo que es de importancia histórica; b) otra que aborda la identificación y la compresión de la continuidad y del cambio histórico; y c) aquella dirigida a identificar la multiplicidad de causas y consecuencias en la historia a través de la conciencia histórica y la toma de perspectiva. Concluimos nuestro capítulo con una serie de orientaciones prácticas dirigidas al profesorado o agentes relevantes en la enseñanza de la historia.

PALABRAS CLAVE: Memoria colectiva; representaciones sociales; historia; enseñanza; competencias educativas.

\section{IMPLICATIONS OF RESEARCH ON COLLECTIVE MEMORY FOR HISTORY EDUCATION}

Copyright: (C) 2018 CSIC. Este es un artículo de acceso abierto distribuido bajo los términos de la licencia de uso y distribución Creative Commons Reconocimiento 4.0 Internacional (CC BY 4.0).

ABSTRACT: Research on collective memory and social representations of history within the field of social psychology may provide guidelines for strengthening meta-cognitive competences in history education. This chapter will review existing literature and empirical research in this field. First, we analyze the processes through which the memories and representations of the past are formed. Next, we present three reasoning competences necessary to be applied in teaching practices: a) a competence focused on determining what is of historical importance; b) a competence that addresses the identification and understanding of the historical continuity and change; c) a competence that aims to identify the multiple causes and consequences in history through historical consciousness and perspective taking. We close our chapter with practical guidelines for history teachers and other relevant ones for history education agents.

KEYWORDS: Collective memory; social representations; history; education; education competencies. 


\section{INTRODUCCIÓN}

La investigación sobre la memoria colectiva y las representaciones sociales del pasado en el campo de la psicología social puede proporcionar reflexiones de interés para el fortalecimiento de las competencias meta-cognitivas en la enseñanza de la historia. Este campo de investigación ha centrado su interés en el modo en el que los grupos sociales preservan y transmiten su herencia a través de las generaciones.

La figura central en la cuestión de la memoria como fenómeno social es Halbwachs (1925/1950), quien la define como la memoria de los miembros de un grupo que reconstruyen su pasado a partir de sus intereses y marco de referencias presentes. Esta memoria asegura la identidad y el valor de un grupo. Halbwachs distingue la memoria de la historia, argumentando que mientras la historia pretende dar cuenta de las transformaciones de la sociedad, la memoria colectiva insiste en asegurar la permanencia y la homogeneidad del grupo. La posibilidad de recordar y rememorar algo del propio pasado sostiene la identidad, en la medida en que es un factor extremadamente importante del sentimiento de continuidad y de coherencia en su reconstrucción de sí mismo (Pollak, 1992).

Halbwachs está influenciado por el concepto de representaciones colectivas de Durkheim y también converge con la perspectiva de las representaciones sociales de Serge Moscovici (1976). Sin embargo, mientras Durkheim hacía referencia a la homogeneidad y al pensamiento dominante en una sociedad, Moscovici rescató la idea de la diversidad social en el interior de los grupos. Para el autor, no hay una memoria sino múltiples memorias, no hay una representación sino múltiples representaciones ligadas a las identidades de los grupos. Por ello, a la hora de desarrollar competencias meta-cognitivas en la enseñanza de la historia, es necesario tener en cuenta esta multiplicidad de visiones, así como el modo en el que se transcienden los intereses grupales para producir memorias inclusivas que confluyan en la construcción de la historia de los pueblos.

\section{REPRESENTACIONES SOCIALES SOBRE EL PASADO, SESGOS Y PROCESOS DE CREACIÓN}

La memoria colectiva o las representaciones sociales sobre el pasado de un grupo social se construyen a partir de imágenes y conocimientos que son elaborados, transmitidos y conservados a través de la comunicación interpersonal e institucional. Estas representaciones sirven para preservar un sentido de continuidad, reforzar una imagen positiva del grupo y cultivar los valores y normas grupales (Pennebaker, Páez y Rimé, 1997). Para ello, es importante tener en cuenta distintos tipos de procesos que determinan el funcionamiento de las representaciones sociales a la hora de reforzar las competencias meta-cognitivas en la enseñanza de la historia (Jodelet, 2011; Lautier, 2001; Tutiaux-Guillon, 2012):

(a) El proceso de anclaje: es el modo en el que una nueva información (en este caso la educación de la historia) se integra en las cosmovisiones pre-existentes. Es decir, se trata del proceso a través del cual la nueva información será interpretada por el alumnado a partir de lo "ya conocido", la experiencia previa, la pertenencia grupal y los valores del propio grupo. Por ejemplo, es sabido que los jóvenes migrantes están menos interesados que sus pares europeos en la historia de las naciones europeas. Asimismo, la juventud musulmana es más crítica sobre temas del holocausto y construyen más analogías entre la segunda guerra mundial y el actual conflicto entre Israel y Palestina (Grever, 2012; Lautier, 2001). El anclaje hace también referencia al proceso de selección y reinterpretación de la información para que se adecue al cuadro cultural como ilustraron los estudios de Barttlet (1932/1973) y Jodelet (2011).

(b) Por su parte, el proceso de objetivación hace referencia a cómo algo abstracto se transforma en algo casi concreto. En el caso que nos ocupa, se ha observado cómo los acontecimientos históricos son objetivados a través de las figuras o personajes históricos ligados al evento, como Colón para hacer referencia al descubrimiento de América o Hitler en relación al nazismo (Lautier, 2001). Es lo que Barttlet denominaba esquematización para referirse al proceso donde se simplifica y concreta la información para adecuarla al marco cultural.

(c) Finalmente, la polifasia cognitiva implica una coexistencia dinámica de distintas modalidades de conocimiento: el tradicional y el nuevo, por un lado, así como el conocimiento "lento", discursivo, lógico o analítico con otro de tipo "rápido", heurístico o mágico (Moscovici, 1976), que podría estar implicado en la superación de las barreras a la conciencia histórica y la toma de perspectiva en la enseñanza de la historia.

\section{PSICOLOGÍA DEL RUMOR Y DISTORSIONES COGNITIVAS EN LA RECONSTRUCCIÓN DEL PASADO}

A estos procesos comunes en la conformación de las representaciones sociales, hay que sumar el papel de los rumores en la reconstrucción de las memorias 
del pasado (Allport y Postman, 1952/1977; Bartlett, 1932/1973), ya que tienen una importancia particular para la enseñanza de la historia. En primer lugar, una historia se narra por medio de rumores o de recuerdos que pasan de un sujeto a otro, simplificando e ignorando muchos elementos de historia. Este proceso se denomina nivelación. Al mismo tiempo, algunos detalles se amplifican o se exageran, convirtiéndolos en centrales para asimilarlos al marco narrativo del recuerdo (asimilación). Consecuentemente, en este recuerdo que ha acentuado los elementos coherentes con la visión general, se agregan detalles coherentes con la visión del grupo adaptándolo a las convenciones, usos, costumbres, valores y estereotipos del grupo (es lo que llamamos convencionalización). Por ejemplo, en general, los manuales de historia destacan especialmente el enfrentamiento de la segunda guerra mundial y el enfrentamiento con los aliados, reconociendo como punto de partida el ataque a Pearl Harbour (condensación y simplificación). No se mencionan las agresiones militares japonesas, los raptos y violaciones de mujeres chinas y coreanas cometidas desde 1931 (Páez y Basabe, 1993).

Cabe destacar que, en la medida en que las representaciones del pasado suelen estar ancladas en acontecimientos históricos que son relevantes para las identidades sociales, en ocasiones, algunos acontecimientos que en principio deberían producir memorias colectivas, son olvidados o silenciados. A veces, los acontecimientos pueden ser tan vergonzantes o culpabilizadores que la mayoría de las personas puedan negarse a hablar de ellos, como en el caso de Dallas, cuyos habitantes negaban el asesinato de Kennedy (Pennebaker y Basanick, 1998). Wagner-Pacifici y Schwartz (1991) sostienen que en ocasiones se reconoce la gravedad de lo ocurrido pero se ignora su sentido social. Incluso, en ocasiones, ocurre lo que Beristain (2000) ha descrito como memorias retorcidas, dispuestas incluso a responsabilizar a las víctimas. Por ejemplo, según algunas encuestas, la mayoría de la población alemana de más de 40 años creía medio siglo después que los judíos fueron en parte responsables del holocausto (Páez y Basabe, 1993).

\section{MEMORIA COLECTIVA, REPRESENTACIONES SOCIALES $Y$ COMPETENCIAS PARA LA ENSEÑANZA DE LA HISTORIA}

La enseñanza de la historia va a estar influenciada por el modo en el que las distintas memorias y representaciones confluyen en la conformación de una narración común. Es necesario tener en cuenta que la enseñanza de la historia a través del sistema edu- cativo en general y de los libros de texto en particular, es solo una de las muchas fuentes para aprender sobre el pasado. Un estudio con estudiantes en Alemania encontró que las novelas históricas y películas se evaluaron como más influyentes que los libros de texto para aprender sobre el pasado. Sin embargo, el estudio también reveló que las clases de historia y los maestros eran más influyentes que el conocimiento trasmitido por los familiares (Von Borries, 1995).

Los cambios en la historiografía tienen, por lo general, una influencia tardía en los libros de historia. Por ejemplo, hasta los años sesenta, los historiadores no prestaron atención a la expulsión de la población palestina en 1948 y elaboraron una narrativa en la que la población palestina habría abandonado la tierra de forma voluntaria. Pasaron veinte años hasta que esta versión fuera incluida en los libros de texto y no sin antes enfrentarse a múltiples resistencias (Bar-Tal, 2014). Aunque los cambios en la enseñanza de la historia tienen un impacto en el modo en el que se construye la memoria y las representaciones sobre el pasado, este proceso suele ser lento en el tiempo. Un estudio realizado en Estados Unidos acerca de las representaciones que la población tenía respecto de la figura de Colón, revela que para la mayoría (85\%), prevalecía aún una imagen neutra que lo caracterizaba en términos de descubrimiento y solo una minoría (3.65\%) pondría de relieve el genocidio indígena que le acompañó (Schuman, Schwartz y D’Arcy, 2005).

Los estudios que se presentan a continuación pretenden visibilizar el modo en el que distintas representaciones y memorias del pasado pueden afectar al aprendizaje de la historia, en particular las competencias de razonamiento necesarias para su aplicación en las prácticas de enseñanza (Seixas, 2012; Grever, 2012).

\section{(1) SIGNIFICACIÓN HISTÓRICA: ACONTECIMIENTOS TRAUMÁTICOS, IMPACTO EMOCIONAL Y RELEVANCIA PARA LA IDENTIDAD GRUPAL}

Entender la significación histórica es la competencia para identificar qué eventos o personajes históricos tienen consecuencias a largo plazo. En concreto, se refiere a por qué nos preocupamos por determinados acontecimientos históricos y no por otros.

\section{(a) Visiones sesgadas: dominación occidental y sesgos sociocéntricos}

Se ha encontrado que, en general, acontecimientos sucedidos en el ámbito europeo o vinculados con América del Norte, como las guerras mundiales, la revolución francesa o la reforma luterana, son los 
eventos que más importantes se consideran (Glowsky, Ellerman, Kromeier, y Andorfer, 2008; Liu et al., 2009; Pennebaker, Páez, y Deschamps, 2006), de forma coherente con lo reflejado en los libros de historia, centrados principalmente en la historia occidental (Tutiaux-Guillon, 2012; López y Carretero, 2012).

Ahora bien, esta disposición se combina con otra tendencia hacia el sociocentrismo: cada país tiende a considerar como más importantes los acontecimientos o personajes históricos de carácter local (Bobowik et al., 2010). Por ejemplo, en España, los participantes calificaron la guerra civil española como el acontecimiento más importante del siglo, mientras que los participantes de Estados Unidos lo hicieron en relación a la guerra civil americana. En general, los países occidentales informaron sobre muy pocos eventos ocurridos fuera de su propia región. El sociocentrismo es también evidente en las nominaciones de personajes históricos importantes. La bibliografía muestra cómo las personas tienden a recordar a personajes históricos del endogrupo. Destaca el caso de Nelson Mandela, quien, aunque fue mayoritariamente valorado de forma positiva, lo fue de forma significativamente más positiva en África que en el resto de los continentes (Liu et al., 2009). Curiosamente, en cierta medida, la misma regla se aplica con relación a los villanos. Por ejemplo, la población española mencionó a Francisco Franco, mientras la portuguesa citó a Antonio Salazar entre los diez personajes más importantes de la historia del mundo.

Sin embargo, el sesgo sociocéntrico no es válido para todas las naciones. Por ejemplo, la evaluación positiva del Che Guevara como personaje histórico latinoamericano, es significativamente mayor en los países europeos y africanos en comparación con los países de América Latina, mostrando que Guevara es más bien un símbolo mundial de la lucha contra la injusticia social que un líder histórico latinoamericano (Pennebaker, Páez y Deschamps, 2006).

\section{(b) Anclaje de la violencia y de pasados históricos traumáticos}

La memoria colectiva conserva los acontecimientos extremos que afectan a un gran número de personas y suelen estar relacionados con cambios importantes en el tejido social o con amenazas importantes para la cohesión nacional (Hilton y Liu, 2008; Pennebaker, Páez y Rimé, 1997). A pesar de que las guerras han producido sólo el $2 \%$ de las muertes durante el siglo XX (Layard, 2005), la gente tiende a destacar el papel de la violencia, las guerras y los líderes militares en la historia del mundo y en sus efectos demográficos catastróficos. Diversos estudios han encontrado la centralidad y el consenso en torno a la violencia como acontecimiento central en las memorias colectivas (Arnoso, Arnoso y Pérez Sales, 2012; Pennebaker, Páez y Deschamps, 2006), mientras que la ciencia y la tecnología, incluyendo la revolución industrial, son secundarios en importancia. En veinticuatro países de América, Europa y Asia, la guerra y la violencia colectiva representaron el $48 \%$ de los acontecimientos recordados. Además, el $45 \%$ de los líderes nombrados, eran conocidos por su papel en distintas guerras o eventos marcados por la violencia colectiva (Liu et al., 2009). El carácter central de guerras y conflictos está ligado al impacto emocional que estos generan. Por ejemplo, acontecimientos como el asesinato de John F. Kennedy, el 11 de septiembre (11-S), los desastres naturales o las crisis financieras, entre otros, tienen un fuerte impacto emocional que se comparte socialmente. Este compartir de emociones intensas como la ira, la tristeza, el miedo y la ansiedad (entre otras) es fundamental en la creación y el mantenimiento de la memoria colectiva (Neal, 1998/2005).

El anclaje de la violencia como un factor principal en las representaciones sociales de la historia es congruente con la historiografía del siglo XIX, donde los historiadores académicos compartieron un enfoque romántico basado en la filosofía idealista alemana, rechazando el principio de las leyes y la causalidad en la historia y centrándose en las descripciones ideográficas de la vida política, de la guerra y los grandes hombres. Por ejemplo, el historiador alemán von Ranke situó los conflictos bélicos como principales agentes de cambio, con el argumento de que solo a través de la guerra una nación se convierte en una nación y afirmando que sin guerras no habría estados (Iggers, Wang y Mukherjee, 2008). Estas representaciones en las que predominan los acontecimientos bélicos son coherentes con su visibilización en los medios de comunicación y los libros de texto históricos, donde, por lo general, los tiempos de guerra reciben mayor cantidad de atención (Pingel, 2000; Zerubavel, 2003).

\section{(c) Identidades sociales y reconstrucción del pasado}

Además, las representaciones sociales del pasado suelen estar ancladas en acontecimientos históricos que son relevantes para las identidades sociales, que son capaces de provocar un cambio social o amenazan la identidad de grupo. Por ello, y tal como se ha señalado, el pasado puede ser silenciado o distorsionado afectando al modo en el que se transmite y se enseña la historia. Por ejemplo, en los países donde las ca- 
tástrofes y las represiones colectivas han tenido lugar (Alemania, Italia, Francia, España, Argentina, Chile o Uruguay, por citar solamente algunos) la respuesta institucional ha sido, al menos durante algunas décadas, la de olvidar y neutralizar lo que ocurrió, no incluyendo en los textos de historia aquellos aspectos que deseaban borrar de la memoria colectiva. Además, el silencio institucional puede operar de la mano de un silenciamiento informal, tanto de los represores como de las víctimas. En el caso de los supervivientes de masacres colectivas, el olvido ha podido aparecer como una forma de evitar la vergüenza por lo sufrido. Los estudios históricos muestran que el olvido y el silencio son reacciones muy frecuentes entre las víctimas de violencia extrema y que la gente prefiere no hablar ni oír hablar sobre las mismas (Wieviorka, 1992). En palabras de Primo Levi (2000), "quien ha sido herido tiende a rechazar el recuerdo para no renovar el dolor, quien ha herido arroja el recuerdo a lo más profundo" (p. 22).

\section{(d) La experiencia histórica y los ciclos del recuerdo}

Por otro lado, es necesario reflexionar acerca del cómo los acontecimientos significativos del pasado están condicionados por el modo en el que la experiencia histórica reciente es accesible para las personas, en tanto está anclada en la experiencia directa y en la memoria comunicativa. Por ejemplo, sabemos que los británicos eran más propensos a recordar la segunda guerra mundial que los estadounidenses, probablemente porque los británicos experimentaron la guerra de forma mucho más directa y personal que los otros (Scott y Zac, 1993). Además de la cercanía espacial, la cercanía temporal en la experiencia histórica también se ha mostrado relevante.

De acuerdo con Manheim (1952), las memorias colectivas dependen en gran parte de la cohorte generacional de pertenencia. Para el autor, más que las bases biológicas o cronológicas, es la localización social lo que identifica a las generaciones, siendo estas formadas por las experiencias compartidas que sus miembros observan. Según Mannheim (1952) "los datos mentales son de importancia sociológica, no solo por su contenido real, sino también porque ello produce que los individuos lo compartan para formar un grupo, (es decir) tienen un efecto socializante" (p. 304). Las personas que comparten un contexto socio-histórico y que toman de la experiencia un conocimiento de tipo similar, forman un grupo social, una unidad generacional. De este modo, Mannheim planteó que las generaciones o cohortes se conformaban como unidades sociales mediante un conjunto de experiencias sociopolíticas compartidas. Cada generación ten- dría una posición social única, basada en experiencias históricas, que la identificaría.

Además, la evidencia empírica ha confirmado que las personas recuerdan mejor los acontecimientos históricos y colectivos experimentados durante la adolescencia o edad adulta temprana. Pennebaker y Basanick (1998) desarrollaron la hipótesis del periodo crítico, explicando que los sucesos, tanto autobiográficos como acontecimientos nacionales o mundiales, ocurridos cuando las personas tenían entre 12 y 25 años, serán más significativos y persistentes en la memoria de las mismas. Schumann y Scott (1989) (en Schuman, Belli, Bischoping, 1997) encontraron que cuando se preguntaba a personas de diversas edades sobre cambios o acontecimientos históricos que les parecían más importantes, hacían referencia a aquellos que ocurrieron al final de su adolescencia o en los primeros años de su juventud. En este sentido se argumenta que este es el periodo de formación de la identidad, de las relaciones íntimas y es un tiempo de gran inestabilidad emocional. Por otro lado, Pennebaker y Basanick (1998) afirman que los sucesos nacionales crearán memorias colectivas más profundas en una sola cohorte de la sociedad que en todos sus demás miembros. Consideran que los eventos, en el momento en que suceden, deben ser también importantes y significativos para la cohorte generacional.

\section{(e) Rituales de conmemoración}

Sin duda, la significación de la historia podrá medirse a través de los modos en los que las memorias son compartidas a través de rituales de conmemoración. Los ritos, las ceremonias y las tradiciones cumplen con el fin de aumentar la cohesión social a través del compartir colectivo de sentimientos, percepciones y recuerdos. Los rituales de conmemoración constituyen formas muy potentes de mantener vivas las memorias colectivas a través del tiempo. Para ello, es importante contar con el apoyo de las instituciones en la promoción de los rituales de recuerdo. Pennebaker y Basanick (1998) han encontrado que suele haber un lapsus de aproximadamente veinticinco años entre la experiencia traumática y la posibilidad de construir monumentos y elaborar abiertamente el pasado. A la hipótesis del periodo crítico, los autores añadieron la hipótesis de la existencia de recursos económicos y el poder social necesario para conmemorar aquellos acontecimientos, así como la tesis de la distancia psicológica para que las emociones negativas o las representaciones conflictivas sobre el pasado desaparezcan y la sociedad se ponga de acuerdo sobre cómo afrontar y validar el recuerdo. 


\section{(f) Sesgo positivista vs. actitudes del presente}

En otro sentido, algunos estudios han encontrado cierta tendencia a evaluar de forma más positiva los acontecimientos históricos más antiguos en comparación con acontecimientos recientes (Bobowik et al., 2010), exagerando, por ejemplo, los aspectos positivos del descubrimiento de América, la revolución francesa o la revolución industrial (entre otros). Evaluar la revolución francesa positivamente sugiere la activación de un sesgo positivista que olvida el terror, las guerras y masacres napoleónicas o, al menos, que el paso del tiempo permite a los individuos y a los grupos reinterpretar los sucesos. Esto es congruente con la tendencia psicológica de reducir al mínimo los estímulos negativos a largo plazo (Taylor, 1991). Los estudios que comparan los recuerdos autobiográficos de las personas en función de su edad han confirmado esta tendencia positivista: una mayor edad se asocia con una valoración más positiva de los recuerdos propios (Laurens, 2002).

Por otro lado, la memoria colectiva o las representaciones sociales del pasado se movilizan para servir a las actitudes y necesidades del presente. Cuando se abre la estructura de oportunidades para los procesos de recuerdo, no se recuperarán todas las experiencias, sino que individuos y colectividades recordarán aquello que sirva para algo en el curso de las acciones presentes. Por ejemplo, en el año 1985 el $30 \%$ de los ciudadanos de Estados Unidos mencionó la segunda guerra mundial como un evento histórico importante; aunque esta se redujo al $20 \%$ en el año 2000 , tras los acontecimientos del 11-S, el porcentaje volvió a ascender al $28 \%$, en una "resurrección" de la segunda guerra mundial como un evento histórico en el contexto de la violencia terrorista internacional (Schuman y Rodgers, 2004).

\section{(g) Memorias en conflicto: silencio u olvido}

Otro punto importante en las dinámicas de la memoria colectiva es el conflicto frecuente entre las memorias oficiales y las transmitidas de forma informal. De acuerdo con Calveiro (2002), la memoria es siempre un relato social que integra voces diversas, donde lo que se busca no es armar un relato único, sino hacer presente la contradicción, la diferencia y la polémica como elementos que permitan la construcción de dimensiones complejas, cuestionando el modo lineal y unívoco de pensamiento. Las memorias y narraciones del pasado implican una moral, señalan una utopía y por tanto vienen acompañadas de un componente ideológico, una dimensión final y una cierta filosofía de la historia (Rivero, Bellelli y Bakhurst, 2000).
Por ejemplo, en España, aunque había tanto personas de derechas como de izquierdas que mencionaban la guerra civil como un hecho central de la historia, las de derechas minimizaban su importancia y gravedad (Páez y Liu, 2010). Los perpetradores directos y vicarios tienden a amortiguar el conflicto ético que genera el uso de la violencia, atribuyendo la responsabilidad de esas acciones al otro grupo, descalifican a las víctimas y minimizan su sufrimiento (Sabucedo, Blanco y de la Corte, 2003). Numerosos estudios muestran la existencia de visiones sesgadas de la realidad, dirigidas a exculpar y minimizar la responsabilidad personal o grupal en los hechos, a favorecer la idea de que el grupo externo es el agresor y la encarnación del mal y el propio es la víctima (Bandura, 1999; Hewstone, Jaspars y Lallje, 1982; Sabucedo, Blanco y de la Corte, 2003). Un estudio realizado en Chile acerca de la memoria colectiva del golpe de estado mostró una década después de la vuelta de la democracia que, aunque se encontraron consensos con relación al reconocimiento de los hechos, las diferencias ideológicas influían en qué y cómo se recordaba el pasado (Manzi, 2006), mientras el $47.3 \%$ evaluaba los hechos como injustificados, la otra mitad le otorgaba alguna o mucha justificación, siendo las personas de derechas quienes más justificaban el golpe.

En el caso de Alemania, la posición oficial o institucional asumió la responsabilidad de la nación en los crímenes de la segunda guerra mundial. Sin embargo, a inicios del siglo XXI, aún un $40 \%$ de la población alemana opinaba que los alemanes fueron espectadores pasivos y el $23 \%$ que fueron víctimas de los nazis (Langenbacher, 2003, en Páez y Liu, 2010). En una línea similar, los nietos de los alemanes que vivieron como adultos en la segunda guerra mundial compartían una visión más positiva del rol de sus abuelos durante la guerra que los abuelos sobre sí mismos (Moller, 2012). Es decir, a pesar de la autocrítica institucional, la opinión pública informal en Alemania rechaza la culpa colectiva y está de acuerdo con el olvido del pasado negativo.

\section{(h) La exclusión de la voz de las mujeres}

Finalmente, una cuestión escasamente abordada en la bibliografía se refiere a la existencia o no de una mirada diferenciada asociada al género en las memorias del pasado. Esta escasez es coherente con la exclusión a la que durante siglos enteros han estado expuestas las voces de las mujeres en la construcción de la historia de la humanidad. Las mujeres podrían considerarse como las grandes olvidadas de 
la historia y también por las investigaciones sobre la memoria colectiva desde una perspectiva anclada en el género. Aun y todo, algunas investigaciones de tipo cualitativo parecen señalar que hombres y mujeres desarrollan habilidades diferentes en lo que concierne a la memoria, habilidades que conducen a prestar más atención a ciertos campos sociales y culturales que a otros y a definir las identidades ancladas en ciertas actividades más que en otras (Jelin, 2002). Las mujeres tienden a recordar la vida cotidiana, la situación económica de la familia, lo que se suponía que debían hacer en cada momento del día, lo que ocurría en sus barrios y comunidades, sus miedos y sentimientos de inseguridad. Por ejemplo, en el caso de las memorias marcadas por la represión política, se ha encontrado que muchas mujeres parecen narrar sus recuerdos en la clave más tradicional del rol de mujer, la de "vivir para los otros", y en ocasiones incluso llegan a "narrar al otro", corriendo la propia identidad. Los testimonios de los hombres se encuentran sin embargo en documentos públicos, en testimonios judiciales y en informes periodísticos. Relatan desde una lógica racional y política, minimizando los aspectos emocionales vinculados a estas experiencias (Jelin, 2002, pp. 108-109). Los estudios con sobrevivientes de la Shoa en relación con las prácticas diferentes en cuanto al modo de hacer públicas las memorias, indican que las narrativas de las mujeres eran menos, entre otras cosas porque hubo menos mujeres que sobrevivieron a los campos de concentración (Glanz, 2001, en Jelin, 2002). En sus relatos, la supervivencia está ligada a la reproducción de los roles aprendidos en la socialización: la limpieza, las habilidades para coser o el cuidado de los otros, que permitieron reinventar los lazos familiares en el interior de los campos (Goldenberg, 1990, en Jelin, 2002).

En el caso de las mujeres secuestradas y forzadas sexualmente para establecer estaciones de servicios sexuales para servir a las tropas japonesas durante la segunda guerra mundial el silencio de su experiencia durante más de cincuenta años fue una constante. La agresión japonesa tuvo éxito en enmudecer a las víctimas (Chizuko, 1999, en Arnoso, Ansaloni, Gandarias y Arnoso, 2012). En el caso de la represión en el cono sur latinoamericano, la realidad parece distinta: han sido las mujeres (madres, abuelas, viudas, etc.) quienes, saliendo de los roles tradicionales de género, han conservado las memorias de la represión, convirtiéndose en un símbolo colectivo, público y político a nivel mundial en la recuperación de la memoria colectiva de los pasados políticos traumáticos recientes (Arnoso, Ansaloni, Gandarias y Arnoso, 2012). Gracias a esos grupos, la memoria puede convertirse en algunas ocasiones en una memoria abierta, en una masa en red que atrae a hacia el sentido de justicia (Jodelet, 2006; Jodelet, 2011). Sus voces implican el reconocimiento y legitimación de otras experiencias además de las dominantes que pueden llevar eventualmente a una transformación del contenido y marco de la memoria social, en la medida en que pueden significar una redefinición de la esfera pública definida de antemano.

\section{(2) IDENTIFICACIÓN Y COMPRESIÓN DE LA CONTINUIDAD Y DEL CAMBIO HISTÓRICO: PERCEPCIONES DE LA HISTORIA}

Además de la significación de la historia, otro elemento relevante de las competencias meta-cognitivas para el proceso de aprendizaje de la historia es la dicotomía entre el cambio o la continuidad histórica, la idea de progreso versus la idea de la repetición, pero también la inclusión de la decadencia o el declive.

\section{(a) Las percepciones de la historia como un proceso lineal y progresista}

Estas percepciones fueron dominantes en los siglos XVIII y XIX, seguramente como resultado del progreso económico y científico de la época. La historia sería comprendida a través de un determinismo económico lícito, tal como ha sido interpretada por el evolucionismo social. Numerosos pensadores (por ejemplo, Kant) apoyaron una interpretación whig de la historia en la que la humanidad avanzaría de la barbarie y la ignorancia hacia la paz, la libertad y la prosperidad. Este tipo de representaciones siguen en la actualidad vigentes, tanto en el cine, la televisión y la retórica política como en los libros de texto de historia (Barton, 2012; Foster, 2012; Tutiaux-Guillon, 2012; Wertsch, 2002). Las encuestas han evidenciado que la visión de la historia como narración de progreso es dominante entre diversas naciones (Páez, Bobowik, Liu, y Basabe, 2015). Además, se ha encontrado que el acuerdo con una representación de la historia ligada al progreso social se asocia con una visión más positiva de las guerras y una evaluación menos negativa de las catástrofes sociales, claramente identificable en el abuso del argumento del progreso como justificador del genocidio contra la población indígena (López y Carretero, 2012).

\section{(b) De la repetición a la decadencia en la historia de la humanidad}

Por otro lado, la mayoría de las culturas antiguas han tenido una concepción circular de la historia, como es el caso de las religiones de la India (pen- 
samiento indio de Vedas) o las teorías cíclicas de la historia desarrolladas en el mundo islámico por Ibn Jaldún quien refería que "nada hay nuevo bajo el sol" (Fontana, 2000; Iggers, Wang y Mukherjee, 2008). También en el mundo occidental las representaciones cíclicas de la historia han tenido sus promotores, algunos de ellos indicando que las civilizaciones tienen una génesis, un desarrollo pleno y un proceso de decadencia que puede lograr que esa civilización muera por completo o que se reinvente y vuelva a crecer expresada de otra manera. Una visión cíclica de la historia podría ser relacionada con la concepción de Vico de la espiral de la historia (1744/1973), quien refirió que la historia tendría tres estadios cíclicos: uno de anarquía y salvajismo; otro de orden, civilización y razón, y un tercero de decadencia y barbarie, todos ellos relacionados con diferentes formas políticas como la teocracia, la aristocracia, la república o la monarquía. Por su parte, Spengler, identificaría esta tendencia cíclica en la vida de las distintas culturas, las cuales también atravesarían una carrera vital predeterminada (juventud, madurez y decrepitud), estando en la actualidad viviendo la vejez de la cultura occidental. El mismo Marx sostenía que la historia siempre se repite dos veces: una vez como tragedia y la segunda vez como comedia (Fontana, 2000).

Así mismo, la teoría crítica post-marxista, bajo las propuestas de sistema-mundo de Wallerstein (2011) propondría que el desarrollo del capitalismo culminaría con una serie de mecanismos que redistribuyen los recursos desde la 'periferia' al 'centro' del imperio, enfrentando las tesis y posiciones ortodoxas que verían en el desarrollo económico del capitalismo un progreso para el conjunto de la humanidad y subrayando que bajo la idea del progreso lo que realmente estaría ocurriendo sería una explotación del centro sobre la periferia y una barbarie invisibilizada por unos medios de comunicación que solo reflejarían el progreso del centro del imperio. Un estudio transcultural reveló que esta mirada cíclica, de ascenso y caída (Hobsbawm, 1995), es apoyada tanto en las culturas orientales como las occidentales, especialmente en el mundo anglosajón, europeo y en las naciones asiáticas (Pingel, 2000). Además, estas creencias están especialmente arraigadas en los países económicamente más desarrollados y en países principalmente individualistas, lo que probablemente refleja las preocupaciones modernas sobre los límites ecológicos y sociales del crecimiento económico (Inglehart, Basañez, Díez-Medrano, Halman y Luijkx, 2004).

\section{(3) TOMA DE PERSPECTIVA EN IDENTIFICAR LAS CAUSAS Y CONSECUENCIAS DEL CAMBIO O LA REPETICIÓN HISTÓRICA}

Finalmente, en el aprendizaje de la historia, es importante la capacidad de identificar las múltiples causas que influyen en el cambio o en la repetición de la historia, así como la toma de perspectiva histórica o la conciencia de que en diferentes épocas existían diferentes mundos culturales (Seixas, 2012).

\section{(a) Las causas y consecuencias del cambio o la repetición histórica}

En la actualidad, la enseñanza laica ha sustituido una enseñanza de tipo religioso que explicaba el desarrollo de la historia asociado a la voluntad de poderes superiores (Seixas, 2012), donde los cambios o las repeticiones estarían sujetos a decisiones divinas. Este enfoque teológico (Bujarin, 1925/1974) ha sido mayoritariamente rechazado en la actualidad en las encuestas con estudiantes universitarios, aunque un tercio de los jóvenes estudiantes migrantes en Francia, así como el $13 \%$ de ascendencia francesa, comparten esta visión de la historia (Tutiaux-Guillon, 2012).

Asimismo, un enfoque centrado en los "grandes hombres", capaces de determinar la dirección principal de la historia (Moscovici, 1985), jugó un papel central en las narrativas nacionalistas del siglo XIX y XX en Europa y en América (Carretero, 2009). A pesar de que los historiadores hayan rechazado esta teoría de la historia, los medios de comunicación han seguido, a través de casos particulares y narrativas individuales, transmitiendo este papel central de personalidades históricas (Moscovici, 1985). El modo en el que los medios de comunicación encuadran las noticias para hacerlas más atractivas desalienta a los espectadores en la atribución de la causalidad de los acontecimientos a los factores sociales (lyengar, 2005). En este sentido, un reciente estudio en treinta y siete países (Hanke et al., 2015) sobre cuarenta personajes de la historia mundial muestra que el mayor consenso entre culturas se manifiesta en torno a recordar a personajes científicos innovadores como Einstein, seguido de figuras humanitarias pacifistas como la madre Teresa de Calcuta y Ghandi. Por otra parte, las figuras negativas obtienen menos consenso, como Hitler, Osaba Bin Laden y Saddam Hussein. Respecto a las diferencias por culturas, los idealistas religiosos y seculares están sobre-representados en los países predominantemente cristianos, mientras los políticos realistas son más frecuentes entre los países preferentemente musulmanes o en los países asiáticos. 
Las guerras como principales agentes del cambio que determinan el curso de la historia fueron enaltecidas por la visión romántica de historiadores académicos del siglo XIX (Iggers, Wang y Mukherjee, 2008) y diversos estudios han mostrado que aún hoy la población considera que las revoluciones o eventos relacionados con la guerra han sido más relevantes que otros para entender los procesos históricos (Liu et al, 2009), lo que sugiere que las personas comparten una visión de la historia como un producto de la violencia (Moscovici, 1985). El darwinismo social, el marxismo y el evolucionismo social también aportaron argumentos para explicar los procesos de la historia. Spencer y otros utilizaron las ideas biológicas de Darwin para apoyar su argumento de que una lucha entre los diferentes pueblos y naciones llevaría a aquellas más fuertes y capaces a gobernar el mundo. La idea de la historia como resultado de la violencia era relevante en el siglo XIX, y el conflicto social y la revolución eran rasgos centrales en la doctrina tanto de Marx como de Engels.

Entrados más en el siglo XX, la perspectiva de la ciencia y la tecnología se integraron en el abordaje de la casuística del desarrollo histórico. Después de la segunda guerra mundial, historiadores económicos como Rostow (1961) hicieron hincapié en la idea de modernización científica y en el desarrollo tecnológico como factor principal de la historia. Confirmando la prevalencia de estas creencias, el $54 \%$ de los encuestados de ochenta y cinco naciones de la World Value Survey acordó que los avances científicos de las últimas décadas suponían una ayuda en el progreso de la humanidad (Inglehart et al., 2004).

\section{(b) Toma de perspectiva: comprensión del pasado y de sus múltiples periodos}

Igualmente es necesario señalar que la competencia sobre la toma de perspectiva histórica también implica la comprensión del pasado en diferentes contextos y la conciencia de la existencia de diferentes períodos históricos (Seixas, 2012). La investigación sobre el recuerdo libre de los acontecimientos históricos ha mostrado cómo la población evalúa los acontecimientos más recientes como históricamente más significativos que los eventos que ocurrieron hace mucho tiempo (Liu et al., 2009; Pennebaker et al., 2006). Asimismo, se ha encontrado un efecto de asimetría entre los eventos y los afectos positivos y negativos, de manera que los sucesos negativos son más fácilmente detectados e influyen más en la percepción, la evaluación y el juicio. Esta tendencia se supone que es adaptativa porque los sucesos negativos son más informativos y requieren reacciones más rápidas que los positivos (Páez et al., 2015). En este sentido, los medios de comunicación se centran más en las noticias negativas (Bar-Tal, 2014).

Teniendo en cuenta estos hallazgos, hay que considerar que las personas usan diferentes tipos de razonamiento y de pensamiento que pueden compartir y funcionar de forma simultánea. Por ello, la enseñanza de la historia debe tener en cuenta que pueden coexistir diferentes concepciones o teorías sobre la evolución y el cambio en la historia. Asimismo, es importante considerar que el desarrollo del aprendizaje histórico es desigual y que un aprendizaje más avanzado o especializado no implica una desaparición de aproximaciones simplistas que pueden reaparecer.

\section{CONCLUSIONES Y RECOMENDACIONES}

La revisión de la bibliografía expuesta sugiere que son varias las competencias meta-cognitivas que pueden promoverse entre el profesorado para mejorar el aprendizaje de la historia en la enseñanza:

(a) el análisis sobre los indicadores que influyen en que los distintos eventos, personajes o informaciones históricas sean significativas y trasciendan en el largo plazo;

(b) la capacidad de identificar y comprender los procesos de cambio, ciclo y declive en la historia de la humanidad;

(c) la capacidad de comprensión de los múltiples factores intervinientes y la toma de perspectiva en el análisis de la historia.

Para el desarrollo de dichas competencias, pueden ser de ayuda los hallazgos realizados por la investigación sobre las memorias colectivas y las representaciones sociales del pasado, sirviendo de guías para un análisis crítico de los relatos históricos que se transmiten en la bibliografía, los medios de comunicación y también los textos de historia. A continuación se presenta una serie de desafíos que emergen como consecuencia de las distorsiones que afectan a dichas representaciones, acompañadas de recomendaciones de buenas prácticas que pueden ser tenidas en cuenta por el profesorado y por los agentes responsables en el diseño de las líneas curriculares para la enseñanza de la historia.

Desafío 1: Los grupos sociales recuerdan, olvidan y reconstruyen el conocimiento del pasado a través de realidades simplificadas que conforman representaciones y memorias en función de las 
necesidades del presente y de sus identidades sociales. Los individuos tendemos a anclar nuestro recuerdo del pasado en lo cercano y conocido. Asimismo, las representaciones dominantes de la historia universal son etnocéntricas y sometidas a la mirada del mundo occidental. Recomendación: se sugiere ampliar la mirada de la historia, buscar más allá de las narrativas dominantes o excesivamente locales (disminuir el socio o etnocentrismo), incluyendo en la enseñanza, las historias de otras latitudes menos conocidas (África, Asia, etc.) que pueden, sin embargo, ofrecer aprendizajes y herencias de interés para el crecimiento comunitario en occidente. Es necesario reflexionar sobre lo que es históricamente significativo y por qué lo es, cuestionando los motivos que amplifican, especialmente a través de los medios de comunicación, ciertos elementos históricos sobre otros. Los monumentos y los rituales son importantes, pero es necesario considerar que a menudo cuentan solo la historia de los vencedores.

Desafío 2: La centralidad de las guerras y los episodios de violencia enfatizan el conflicto intergrupal en el desarrollo de la humanidad. La objetivación en personajes históricos impide la inclusión de factores de tipo socioestructural y de proceso. Recomendación: se debe evitar normalizar la centralidad de la violencia y el conflicto como forma del progreso. Esta centralidad desplaza la relevancia que otros procesos históricos de carácter positivo puedan tener para el desarrollo de la humanidad. Así, parece conveniente poner de relieve que existen dinámicas capaces de promover cambios colectivos y que además lo hagan a través de emociones colectivas positivas. Los lideres o personajes históricos son relevantes, pero no son capaces de absorber la complejidad de los procesos históricos.

Desafío 3. Muchas memorias han sido silenciadas u olvidadas. Las emociones negativas han jugado un papel importante en ese silenciamiento. Además, existen personajes y eventos históricos mejor recordados y otros más olvidados. Los textos son una fuente importante para la enseñanza, pero no la única. Recomendación: Es necesario incluir en los libros de texto, en las aulas y en los contenidos transmitidos por los medios de comunicación las narrativas silenciadas, especialmente en contextos post conflicto. Hace falta destacar el papel de los supervivientes y sus descendientes para la preservación de la memoria colectiva de hechos de violencia colectivos (guerras, genocidios y violaciones de derechos humanos). Las emociones negativas pueden promover la negación o el silenciamiento ante sucesos vergonzantes para el propio grupo que retrasen la inclusión de ciertas memorias en los libros oficiales (especialmente cuando la violencia viene del estado), por lo que es necesario combinarlos con otros recursos documentales, como las narrativas testimoniales, entre otros. Además, aunque las emociones negativas pueden inducir al silencio, también pueden activar la necesidad de reparar el pasado a través del reconocimiento del daño cometido y redimir así la culpa o la vergüenza asociada a los hechos. Liberar las emociones negativas puede ser de utilidad para transformar el dolor en una imagen más positiva del endogrupo.

Desafío 4: Existe una enorme discrepancia en la reconstrucción del pasado en función del género implicado. La voz de las mujeres ha sido sistemáticamente negada y eso supone una construcción de la historia realizada sin al menos la mitad de la población. Recomendación: Es urgente recuperar las narrativas de las mujeres en la inclusión de la historia. Quizás, sus memorias y representaciones del pasado pudieran proporcionar una mirada completamente distinta de lo que es significativo en la historia de la humanidad. Frente a la centralidad del conflicto en las memorias tradicionales, las memorias de las mujeres están cargadas de recuerdos dirigidos de forma sistemática a la inclusión del otro.

Desafío 5: Todos los tipos de sesgos revisados en este capítulo (el anclaje en la violencia, el socio-centrismo o etno-centrismo, sesgo positivista, sesgos generacionales, el anclaje en la experiencia personal, etc.) impiden la toma de perspectiva histórica o entender que la continuidad histórica tiene diferentes facetas. Recomendación: Es imprescindible fortalecer la competencia de toma de perspectiva en el análisis histórico del pasado. Eso puede ser posible a través de: 1 ) destacar la importancia de factores explicativos del recorrido de la historia alternativos y 2) tomar en cuenta de diferentes maneras de entender la temporalidad (visión lineal y de progreso, circular y cíclica, visión crítica de la explotación del centro sobre la periferia). De esa manera, el profesorado de historia también puede respetar la diversidad cultural del aula, con sus diferentes intereses en función de las identidades sociales diversas (étnicas, religiosas etc.) y diferentes maneras de entender la historia (p.ej. como avance o como un ciclo). 


\section{AGRADECIMIENTOS}

Este trabajo ha sido posible gracias a las ayudas de investigación del Ministerio de Economía, Industria y Competitividad (PSI2011-26315), del gobierno vas- co (IT-666-13) y del proyecto europeo COST Action IS1205 Social psychological dynamics of historical representations in the enlarged European Union.

\section{BIBLIOGRAFÍA}

Allport, G. y Postman, L. (1952/1977). Psicología del rumor. Buenos Aires: Pléyade.

Arnoso, M., Ansaloni, S., Gandarias, I., y Arnoso, A. (2012). Mujeres jujeñas y sobrevivientes: narrativas del pasado represivo (1976-1983) argentino, consecuencias psicosociales y creencias acerca de la reparación. Revista mexicana de ciencias políticas y sociales, 57 (214), pp. 141-161.

Arnoso, M., Arnoso, A., y Pérez-Sales, P. (2012). Representaciones sociales del pasado: la dictadura militar argentina en la memoria colectiva. Revista de Psicología Social, 27, (3), pp. 259-272. https:// doi.org/10.1174/021347412802845540

Bandura, A. (1999). A social cognitive theory of personality. En Pervin, L. y John, O. (eds.). Handbook of personality. New York: Guilford Publications, pp. 154-196.

Bar-Tal. D. (2014). Intractable Conflicts: Socio-Psychological Foundations and Dynamics. Cambridge: Cambridge University Press.

Bartlett, F. C. (1932/1973). Los factores sociales del recuerdo. En Proshansky, H. y Seidenberg, B. (eds.). Estudios básicos de psicología social. Madrid: Tecnos.

Barton, K. C. (2012). School History as a resource for construction identities. En Carretero, M., Asensio, M. y RodríguezMoneo, M. (eds.). History Education and the Construction of National Identities. Charlotte, NC: Information Age Publishing, pp. 93-108.

Beristain, C. M. (2000). Justicia y reconciliación: El papel de la verdad y la justicia en la reconstrucción de sociedades fracturadas por la violencia. Bilbao: Hegoa.

Bobowik, M. Páez, D., Liu, J. H., Espinosa, A., Techio, E., Zubieta, E., y Cabecinhas, R. (2010). Psychosocial bases of a "culture of war": Beliefs about History, the Meaning of Second World War and willingness to fight in Portuguese and Spanish speaking cultures. Revista de Psicología, 28, pp. 111-146.

Bujarin, N. (1925/1974). Teoría del materialismo histórico. Madrid: Siglo XXI.
Calveiro, P. (2002). Poder y desaparición. Los campos de concentración en Argentina. Buenos Aires: Editorial Colihue.

Carretero, M. (2009). Identidad nacional y enseñanza de textos históricos: una hipótesis explicativa. Seminario Internacional. Textos escolares de historia y ciencias sociales. Santiago de Chile: Ministerio de Educación de Chile, pp. 70-77.

Fontana, J. (2000). La historia de los hombres. Barcelona: Crítica.

Foster, S. (2012). Re-thinking History Textbooks in a Globalized World. En Carretero, M., Asensio, M. y Rodríguez-Moneo, M. (eds.). History Education and the Construction of National Identities. Charlotte, NC: Information Age Publishing, pp. 49-62.

Glowsky, D., Ellermann, H., Kromeier, K., y Andorfer, V. (2008). A Global Collective Memory? Results from a Quantitative Pilot Study. Comparativ, 2, pp. 99-115.

Grever, M. (2012). Dilemmas of Common and Plural History: Reflections on History Education and Heritage in a Globalizing World. En Carretero, M., Asensio, M. y Rodríguez-Moneo, M. (eds.). History Education and the Construction of National Identities. Charlotte, NC: Information Age Publishing, pp. 93-108.

Halbwachs, M. (1925/1950). Les cadres sociaux de la mémoire. Paris: Presses Universitaires de France.

Hanke, K., Liu, J. H., Sibley, C. G., Paez, D., Gaines Jr, S. O., Moloney, G. y Cabecinhas, R. (2015). "Heroes" and "Villains" of World History across Cultures. PlosOne, 10 (2), e0115641. https://doi. org/10.1371/journal.pone.0115641

Hewstone, M., Jaspars, J., y Lalljee, M. (1982). Social representations, social attribution and social identity: The intergroup images of 'public' and 'comprehensive' schoolboys. European Journal of Social Psychology, 12 (3), pp. 241-269. https://doi.org/10.1002/ ejsp.2420120302

Hilton, D. J. y Liu, J. H. (2008). Culture and intergroup relations. The role of social representations of history. En Sorrentino, R. y Yamaguchi, D. (eds.). Handbook of Motivation and Cognition Across Cultures. New York: Guilford, pp. 343-368. https://doi.org/10.1016/B978-0-12373694-9.00015-5

Hobsbawm, E. (1995). Historia del siglo XX. Barcelona: Crítica.

Iggers, G. G., Wang, Q. E. y Mukherjee, M. (2008). A Global History of Modern Historiography. Harlow: Pearson.

Inglehart, R., Basañez, M., Diez-Medrano, J., Halman, L. y Luijkx, R. (2004). Human Beliefs and Values. Mexico: Siglo XXI.

Iyengar, S. (2005). Speaking of Values: the Framing of American Politics. The Forum, 3 (3), Article 7. Disponible en: http:// www.bepress.com/forum/vol3/iss3/art7

Jelin, E. (2002). Los trabajos de la memoria. Madrid: Siglo XXI.

Jodelet, D. (2006). Représentations sociales. En Mesure, S. y Savidan, P. (dirs.). Dictionnaire des sciences humaines. Paris: Presses Universitaires de France, pp. 1003-1005.

Jodelet, D. (2011). Contributions from the Social Representations Approach to the Field of Education. Espacios en Blanco Serie indagaciones, 21 (1), pp. 133-154.

Laurens, S. (2002). La nostalgie dans l'élaboration des souvenirs. En Laurens, S. y Roussiau, N. (eds.). La mémoire sociale. Identités et représentations sociales. Rennes: Presses Universitaires de Rennes, pp. 259-268.

Lautier, N. (2001). Psychosociologie de l'éducation. Regard sur la situation d'enseignement. Paris: Armand Colin.

Layard, R. (2005). La Felicidad. Madrid: Taurus.

Levi, P. (2000). Si esto es un hombre. Barcelona: Muchnik.

Liu. J. H., Páez, D., Slawuta, P., Cabecinhas, R., Techio, E., Kokdemir, D., Sen, R., Vincze, O., Muluk, H., Wang, F. y Zlobina, A. (2009). Representing World History in the $21^{\text {st }}$ Century: The Impact of 9/11, the Iraq War, and the Nation-State on 
Dynamics of Collective Remembering. Journal of Cross-Cultural Psychology, 40 (4), pp. 667-692. https://doi. org/10.1177/0022022109335557

López, C. y Carretero, M. (2012). Commentary: Identity Construction and the Goals of History Education. En Carretero, M., Asensio, M. y Rodríguez-Moneo, M. (eds.). History Education and the Construction of National Identities. Charlotte, NC: Information Age Publishing, pp. 93-108.

Mannheim, K. (1952). The sociological problem of generations. En Kecskemeti, P. (ed.). Essays on the Sociology of Knowledge. London: Routledge, pp. 276-322.

Manzi, J. (2006). La memoria colectiva del golpe de estado en Chile. En Carretero, M., Asensio, M. y Rodríguez-Moneo, M. (eds.). History Education and the Construction of National Identities. Charlotte, NC: Information Age Publishing, pp. 297-322.

Moller, S. (2012). Are Family Recollections and Obstacle to History Education? En Carretero, M., Asensio, M. y RodríguezMoneo, M. (eds.). History Education and the Construction of National Identities. Charlotte, NC: Information Age Publishing, pp. 281-296.

Moscovici, S. (1976). La psychanalyse, son image et son public. Paris: Presses Universitaires de France.

Moscovici, S. (1985). L'Age des foules. Un traité historique de psychologie des masses. Bruxelles: Les Éditions Complexe.

Neal, A. G. (1998/2005). National Trauma and Collective Memory. Major Events in the American Century ( $2^{\text {nd }}$. ed.). Armonk, NY: M. E. Sharpe.

Páez, D. y Basabe, N. (1993). Trauma político y memoria colectiva: Freud, Halbwachs y la psicología política contemporánea. Revista de Psicología Política, 6 , pp. 7-34.

Páez, D., Bobowik, M., Liu, J. H. y Basabe, N. (2015). Shared beliefs about world history and cultural context: a theoretical review and a collective-level analysis. En Ch. Stone y L. Bietti (eds.). Contextualizing Human Memory: An interdisciplinary approach to understanding how individuals and groups remember the past. Psychology Press.

Páez, D. y Liu, J. H. (2010). Collective Memory of Conflicts. En D. Bar-Tal (ed.). In- tergroup Conflicts and Their Resolution: A Social Psychological Perspective. New York: Psychology Press.

Pennebaker J. W. y Basanick, B. (1998). Creación y mantenimiento de memorias colectivas. En Páez, D., Valencia, J., Pennebaker, J., Rimé, B. y Jodelet, D. (eds.). Memorias colectivas de procesos culturales y políticos. Bilbao: Universidad del País Vasco. Servicio Editorial, pp. 31-47.

Pennebaker, J. W., Páez, D. y Deschamps, J. C. (2006). The Social Psychology of History: Defining the most important events of the last 10,100, and 1000 years. Psicología Política, 32, pp. 15-32.

Pennebaker, J. W., Páez, D. y Rimé, B. (eds.) (1997). Collective Memory of Political Events: Social Psychological Perspectives. Mahwah, NJ: Erlbaum.

Pingel, F. (2000). The European home: representations of $20^{\text {th }}$ century Europe in history textbooks. Council of Europe Publishing.

Pollak, M. (1992). Memória e identidade social. Estudos Históricos, 5 (10), pp. 200-212.

Rivero, R., Bellelli, G. y Bakhurst, D. (2000). Memoria colectiva e identidad nacional. Madrid: Biblioteca Nueva.

Rostow, W. W. (1961). Las etapas del crecimiento económico. Un manifiesto no comunista. México: Fondo de Cultura Económica.

Sabucedo, J. M., Blanco, A. y de la Corte, L. (2003). Beliefs which legitimize political violence against the innocent. Psichote$m a, 15$ (4), pp. 550-555.

Schuman, H., Belli, R. F. y Bischoping, K. (1997). The generational basis of historical knowledge. En Pennebaker, J. W., Páez, D. y Rimé, B. (eds.). Collective Memory of Political Events: Social Psychological Perspectives. Mahwah, NJ: Erlbaum, pp. 47-77.

Schuman, H. y Rodgers, W. L. (2004). Cohorts, Chronology and Collective Memories. Public Opinion Quarterly, 68, pp. 217-254. https://doi.org/10.1093/ poq/nfh012

Schuman, H., Schwartz, B. y d'Arcy, H. (2005). Elite revisionists and popular beliefs: Cristopher Columbus, hero or villain? Public Opinion Quarterly, 69, pp. 2-29. https://doi.org/10.1093/ poq/nfi001
Scott, J. y Zac, L. (1993). Collective memories in Britain and the United States. Public Opinion Quarterly, 57 (3), pp. 315-351. https://doi.org/10.1086/269378

Seixas, P. (2012). Indigenous Historical Consciousness: An Oxymoron or a Dialogue. En Carretero, M., Asensio, M. y Rodríguez-Moneo, M. (eds.). History Education and the Construction of $\mathrm{Na}$ tional Identities. Charlotte, NC: Information Age Publishing, pp.125-138.

Taylor, S. (1991). Asymmetrical Effects of Positive and Negative Events: The Mobilization-Minimization Hypothesis. Psychological Bulletin, 110 (1), pp. 67-85. https:// doi.org/10.1037/0033-2909.110.1.67

Tutiaux-Guillon, N. (2012). A Traditional Frame for Global History: The Narrative of Modernity in French Secondary School. En Carretero, M., Asensio, M. y Rodríguez-Moneo, M. (eds.). History Education and the Construction of $\mathrm{Na}$ tional Identities. Charlotte, NC: Information Age Publishing, pp. 109-124.

Vico, G. (1744/1973). Principios de una ciencia nueva sobre la naturaleza común de las naciones. Buenos Aires: Aguilar Argentina.

Von Borries, B. (1995). Le Siècle des Découvertes dans les livres scolaires de langue allemande. En Pérez-Siller A. (ed.) La Découverte de l'Amérique. Paris: L'Harmattan.

Wagner-Pacifici, R. y Schwartz, B. (1991). The Vietnam Veterans Memorial: Commemorating a Difficult Past. American Journal of Sociology, 97 (2), pp. 376420. https://doi.org/10.1086/229783

Wallerstein, I. M. (2011). El moderno sistema mundial III. La segunda era de gran expansión de la economía-mundo capitalista, 1730-1850. México: Siglo XXI.

Wertsch, J. V. (2002). Voices of collective remembering. Cambridge: Cambridge University Press. https://doi. org/10.1017/CBO9780511613715

Wieviorka, A. (1992). Déportation et génocide. Entre la mémoire et l'oubli. Paris: Plon.

Zerubavel, E. (2003). Collective Memory and the Social Shape of the Past. Time Maps. Chicago: The University of Chicago Press. https://doi.org/10.7208/chicago/9780226924908.001.0001 